\title{
Sterbe- und Suizidhilfe in der Schweiz: Was gibt es Neues? \\ Andreas Eicker ${ }^{1}$
}

\section{Deutscher "Sterbetourismus" - Handlungs- bedarf für die Schweiz?}

$\int$ er im April 2006 vom Eidgenössischen Justiz- und Polizeidepartement (EJPD) vorgelegte Bericht „Sterbehilfe und Palliativmedizin - Handlungsbedarf für den Bund?" weist darauf hin, dass „Politik und Öffentlichkeit wegen der Missbrauchsgefahren" im Bereich „Sterbetourismus“ derzeit den „grössten [gesetzgeberischen] Handlungsbedarf" sehen ${ }^{2}$. Die Suizidhilfe gegenüber Personen mit Wohnsitz im Ausland (sog. "Sterbetourismus") wird in der Schweiz hauptsächlich von der Suizidhilfeorganisation DigNITAS organisiert und durchgeführt ${ }^{3}$. Während im Jahr 2000 nur 3 Personen aus dem Ausland die Hilfe von DigNITAS in Anspruch nahmen, waren es drei Jahre später schon 91 und im Jahr 2004 immerhin noch 88 Personen ${ }^{4}$. Die Organisation zählt momentan ca. 4.500 Mitglieder in nicht weniger als 52 Ländern, wobei die grösste Gruppe der Mitglieder aus Deutschland stammt ${ }^{5}$. Dignitas hat nach eigenen Angaben seit der Gründung im Jahr 1998 bis zum 15. September 2005 insgesamt 453 Freitodbegleitungen durchgeführt, von denen 253 Personen aus Deutschland stammten ${ }^{6}$. Letzteres mag erklären, warum am 26. September 2005 gerade in Hannover der Verein Dignitas-DeuTsCHLAND als Tochtervereinigung der schweizerischen Suizidhilfe-Organisation gegründet wurde. Bis zum verfahrensgerechten Abschluss eines Streits um den Namen mit der „Deutschen Interessengemeinschaft für Verkehrsunfallopfer e.V. dignitas" operiert DigniTAS-DEUTSCHLAND unter der Bezeichnung DigniTATE.

In der Schweiz werden die begleiteten Suizide ausländischer Sterbewilliger in einer von DiGNiTAS angemieteten Wohnung in Zürich und seit 2004 vorübergehend auch in einem Haus im Kanton Aargau durchgeführt. Nach Aussagen des Generalsekretärs von DigNITAS, LUDWIG A. MinELLI, findet stets ein Gespräch zwischen einem Sterbebegleiter und dem Suizidwilligen statt. Ein Vertrauensarzt der Organisation verschreibt ihm, nachdem er den Sterbewilligen gesehen hat, ein todbringendes Betäubungsmittel. Dieses wird ihm vom Begleiter in Abwesenheit des Arztes zur Verfügung gestellt. Das Betäubungsmittel wird dem Suizidwilligen jedoch nie alleine, d.h. ohne den Begleiter überlassen. Letzterer erhält pro Fall $500 \mathrm{Fr}$. Der Suizidwillige zahlt für die Suizidbegleitung insgesamt 2000 Fr. an Dignitas.

Jeder Suizid wird als nicht natürlicher Todesfall der zuständigen Behörde gemeldet. Die Straf- untersuchungsbehörden klären ab, ob es sich um einen Suizid handelt oder nicht. Voraussetzung dafür ist, dass die verstorbene Person die tatsächliche Herrschaft über das Geschehen besass. Es wird zudem geprüft, ob sowohl die Willensbildung als auch die Willensäusserung ohne Druck durch Dritte zustande gekommen sind. Wird ein Suizid positiv festgestellt, werden auch die übrigen Tatbestandsmerkmale von Art. 115 StGB geprüft. Der Suizidhelfer muss frei von „selbstsüchtigen Beweggründen" gehandelt haben, ansonsten macht er sich strafbar.

Der wesentliche Grund für den Sterbetourismus von Deutschland in die Schweiz liegt in der unterschiedlichen gesetzlichen Regelung der Suizidhilfe. Mit der Strafbestimmung in Art. 115 („Verleitung und Beihilfe zum Selbstmord“) gilt das schweizerische Regelwerk im Verhältnis zum deutschen Rechtssystem als liberal. Gemäss Art. 115 wird nur bestraft, „Wer aus selbstsüchtigen Beweggründen jemanden zum Selbstmorde verleitet oder ihm dazu Hilfe leistet". Diese Regelung hat abschliessenden Charakter. In Deutschland sind Anstiftung und Beihilfe zum Selbstmord zwar nicht speziell unter Strafe gestellt und die Teilnahme an einem Suizid ist strafrechtsdogmatisch mangels strafbarer Haupttat nicht strafbar. Doch kann unter gewissen Umständen jemand, der eine Garantenstellung innehat und einen Suizid nicht verhindert, wegen dieser Unterlassung zur Verantwortung gezogen werden.

Der Schweizer Bundesrat hat am 31. Mai dieses Jahres vom erwähnten „Sterbehilfe-Bericht” des EJPD Kenntnis genommen. Gestützt auf diesen Bericht „empfiehlt” der Bundesrat „dem Parlament, auf eine Revision der einschlägigen Bestimmungen des Strafgesetzbuches sowie auf den Erlass eines Gesetzes über die Zulassung und Beaufsichtigung von Suizidhilfeorganisationen zu verzichten", was auch einen Verzicht auf die Überwachung des Sterbetourismus bedeutet. Diese Haltung ist schon angesichts des in der Öffentlichkeit offenbar erkannten Handlungsbedarfs in Sachen Sterbetourismus bemerkenswert; zudem aber auch deshalb, weil sich der Bundesrat in seinem aus dem Jahr 2000 datierenden Bericht zum „Postulat Ruffy”, welches auf das Jahr 1994 zurückgeht, noch deutlich „für eine ausdrückliche Regelung der passiven und der indirekten aktiven Sterbehilfe" im Strafgesetzbuch ausgesprochen und nur „eine gesetzliche Regelung der direkten aktiven Sterbehilfe" abgelehnt hat ${ }^{\text {. }}$.

Die Entwicklung, die zu diesem „Sinneswandel“ des Bundesrats und zu seiner „Empfehlung“ mit Blick auf den Sterbetourismus geführt hat, wird im Folgenden unter Einbezug der die jeweilige Position tragenden Argumente nachgezeichnet, um den aktuellen Stand der Debatte in der Schweiz zu bestimmen ${ }^{8}$. Dieses kann jedoch nur dann sinnvoll geschehen, wenn zuvor Klarheit über die im Zusammenhang mit der so genannten „Sterbehilfe” verwendeten Begriffe und über die derzeit geltende Rechtslage in der Schweiz geschaffen wird. Dabei wird auch versucht, die aktuelle deutsche Debatte ${ }^{9}$ um den Ende 2005 vorgelegten Alternativentwurf-Sterbebegleitung (AE$\mathrm{StB})^{10}$ aufzugreifen. Letzteres kann hier jedoch schon aus Raumgründen - nur mit wenigen Hinweisen geschehen.

\section{Begriffliche Abgrenzung und rechtliche Einordnung}

Bei den vom Begriff Sterbehilfe (1.) erfassten Tathandlungen handelt es sich ausschliesslich um Fremdtötungen, also solche, bei denen die Tatherrschaft nicht der Sterbewillige selbst hat, sondern stets ein Dritter. Die Sterbehilfe ist insofern von der Suizidhilfe (2.) zu unterscheiden, bei der die Herrschaft über das Tatgeschehen bei der sterbewilligen Person liegt ${ }^{11}$.

\section{Sterbehilfe}

a) Die direkte aktive Sterbehilfe bezeichnet die gezielte Tötung eines anderen Menschen zur Verkürzung von dessen Leiden, z.B. durch Beibringen einer tödlichen Injektion oder Infusion. Diese Form der „Hilfe” zum Sterben ist nach schweizerischem Recht strafbar ${ }^{12}$, es handelt sich strafrechtsdogmatisch um eine Fremdtötung im Sinne der Art. 111 ff. ${ }^{13}$. Zum Teil wird eine Lockerung des absoluten Verbots aktiver Sterbehilfe mit Rücksicht auf den nahen Tod und das Selbstbestimmungsrecht des Sterbenden sowie die tägliche Praxis in den Spitälern allerdings als „aufrichtiger” empfunden ${ }^{14}$. Liegt der Fremdtötung ein „ernsthaftes und eindringliches Verlangen" des Sterbewilligen zugrunde und geschieht sie aus „achtenswerten Beweggründen"15, so wird diese Form der Tötung gemäss Art. 114 gegenüber der vorsätzlichen Tötung nach Art. 111 privilegiert behandelt ${ }^{16}$. Ausdruck findet dies im Strafrahmen, der für die Tötung auf Verlangen „nur“ Gefängnisstrafe ${ }^{17}$ androht, während die vorsätzliche Tötung mit Zuchthausstrafe ${ }^{18}$ bedroht ist ${ }^{19}$.

Der AE-StB 2005 hält an der ausnahmslosen 
Strafbarkeit von Massnahmen der aktiven (direkten) Sterbehilfe fest. „Mit der uneingeschränkten Beibehaltung des strafrechtlichen Verbots aktiver Sterbehilfe” soll „jeder Ansatz zur Durchbrechung des Fremdtötungsverbots vermieden werden" ${ }^{20}$. Diese Position erscheint vertretbar, wenn zugleich die Bereitschaft bestünde, Extremfällen, die auch palliativmedizinisch nicht beherrschbare Leiden für den $\mathrm{Pa}$ tienten bedeuten, mit dem rechtfertigenden Notstand (§ $34 \mathrm{dStGB})$ Rechnung zu tragen ${ }^{21}$.

b) Mit der indirekten aktiven Sterbehilfe ist das Verabreichen von schmerzlindernden Mitteln gemeint, die als - eigentlich unerwünschte Nebenfolge der palliativen (schmerzlindernden) Behandlung eine Verkürzung der (Über-) Lebensdauer bewirken ${ }^{22}$. Da durch indirekte aktive Sterbehilfe der Todeseintritt beschleunigt wird, ist nach allgemeinen Kausalitätsregeln eigentlich eine strafbare Fremdtötung gemäss der Art. 111 ff. gegeben. Nach h.M. ist jedoch die Zulässigkeit der indirekten aktiven Sterbehilfe aus dem Heilauftrag und damit aus der Behandlungspflicht des Arztes abzuleiten. Letzterer ist nicht nur zur Verbesserung des Gesundheitszustandes seines Patienten, sondern auch zur Linderung von dessen Leiden verpflichtet. Er handelt insofern gerechtfertigt im Sinne von Art. $32^{23} 24$, danach ist die Tat, die eine Berufspflicht gebietet, kein Verbrechen oder Vergehen ${ }^{25}$. Gemäss der medizinisch-ethischen Richtlinien der Schweizerischen Akademie der Medizinischen Wissenschaften (SAMW) steht der Arzt ausdrücklich in der Pflicht, Schmerz und Leiden zu lindern, auch wenn dies im Einzelfall zu einer Verkürzung der Lebensdauer führen sollte ${ }^{26}$. Nach anderer Auffassung entfällt schon die objektive Erfolgszurechnung, da der Täter angesichts des sozial anerkannten Gebots, unerträgliche Schmerzen des Sterbenden zu lindern und damit dessen Lebensqualität zu erhöhen, kein rechtlich unerlaubtes Risiko schafft, wenn mit der Schmerzbehandlung zugleich eine Verkürzung der Lebensdauer verbunden ist ${ }^{27}$. Nach wiederum anderer Ansicht ist die Rechtfertigung Ausdruck einer verfassungsrechtlichen Güterabwägung. Danach können das Selbstbestimmungsrecht des Patienten und das Verbot der unmenschlichen Behandlung die Lebenserhaltungspflicht des Arztes überwiegen ${ }^{28}$. Entscheidend für die Straflosigkeit ist nach verbreiteter Auffassung das der Tathandlung zugrunde liegende Handlungsziel. Subjektiv muss der Täter danach die Linderung eines unerträglichen Leidens beabsichtigen, auch wenn dies den Sterbevorgang beschleunigt ${ }^{29}$. Da es sich aber nach den dogmatischen Regeln des Strafrechts auf jeden Fall um eine Fremdtötungshandlung handelt, darf die palliative Behandlung mit lebensverkürzender Wirkung nur als „ultima ratio" eingesetzt werden, wenn es keine weniger folgenschwere Schmerzbehandlung mehr gibt $^{30}$. c) Von passiver Sterbehilfe wird gesprochen, wenn entweder auf lebenserhaltende Massnahmen verzichtet wird oder solche Massnahmen abgebrochen werden. Angesprochen ist damit beispielsweise der Verzicht auf bzw. der Abbruch von künstliche(r) Beatmung und Nahrungsoder Arzneimittelzufuhr. Der Schwerpunkt des Verhaltens, das hier strafrechtlich relevant werden könnte, liegt auf dem Behandlungsverzicht, also auf einem Unterlassen von Behandlungsmassnahmen trotz bestehender Interventionsmöglichkeiten ${ }^{31}$. Dies wird auch bei einem Abbrechen lebenserhaltender Massnahmen angenommen ${ }^{32}$, welches eigentlich (auch) als aktives Tun gewertet werden könnte ${ }^{33}$. Der Schwerpunkt des Handelns liegt jedoch nicht im Vorgang des Abbrechens, sondern im nichtweiter-Behandeln, also in einem Unterlassen ${ }^{34}$. Erfolgt der Behandlungsverzicht auf ausdrücklichen Wunsch des Sterbewilligen, kommt eine Strafbarkeit des Arztes schon deshalb nicht in Betracht, weil jeder Behandlungsbeginn bzw. jedes Fortsetzen der Behandlung die Einwilligung des Patienten voraussetzen würde. Fehlt diese, ist der ärztliche Eingriff eigenmächtig und damit rechtswidrig. Anders formuliert macht sich der Arzt bei einer Behandlung ohne Patienteneinwilligung strafbar ${ }^{35}$, nicht aber wenn er auf diese - wegen fehlender Einwilligung - verzichtet oder sie abbricht. Kann die betroffene Person mangels Urteilsfähigkeit nicht mehr in die Behandlung einwilligen bzw. auf sie verzichten, dann darf auf ihre Patientenverfügung zurückgegriffen werden ${ }^{36}$. Liegt kein schriftlich oder mündlich formulierter ausdrücklicher Behandlungsverzicht vor, dann hat der Arzt seine Entscheidung am mutmasslichen Willen der betroffenen Person auszurichten ${ }^{37}$. Bei Unmündigen gilt grundsätzlich der Wille des gesetzlichen Vertreters. Liegt kein ausdrücklicher Behandlungsverzicht vor und kann auch ein mutmasslicher Behandlungsverzicht nicht ermittelt werden, dann kann sich der Arzt darauf berufen, dass eine Behandlung mit Blick auf die Menschenwürde unzumutbar ist, weil weder eine Verbesserung der Gesundheit noch eine Leidenslinderung bewirkt werden $\mathrm{kann}^{38}$. Der Sterbewunsch geniesst in diesem Bereich auf Grund des Selbstbestimmungsrechts (Art. 10 Abs. 2 Schweizer Bundesverfassung) des Patienten auch grundrechtlichen Schutz ${ }^{39}$.

Der AE-StB 2005 wendet sich gegen die Begriffe „indirekte” und „passive” Sterbehilfe. Der vorgeschlagene Begriffswechsel sieht vor, statt dessen von „leidenslindernden Massnahmen” (§ 214a AE-StB) und von „Beenden, Begrenzen oder Unterlassen lebenserhaltender Massnahmen" (§ 214 AE-StB) zu sprechen ${ }^{40}$. In inhaltlicher Hinsicht ist für beide Gesetzesvorschläge $^{40}$ die Tendenz zur Prozeduralisierung des Rechts bemerkenswert. Insbesondere bezüglich der Durchführung der indirekten Sterbehilfe ("leidenslindernde Massnahmen") und mit
Blick auf die Beurteilung des mutmasslichen Willens bei der passiven Sterbehilfe („Beenden, Begrenzen oder Unterlassen lebenserhaltender Massnahmen") werden materiellrechtliche Elemente mit prozeduralen verbunden. Letztere sind in einem AE-Sterbebegleitungsgesetz ausgearbeitet.

\section{Suizidhilfe}

Im Gegensatz zu den von der Sterbehilfe erfassten Konstellationen liegt die Tatherrschaft bei der $\mathrm{Su}$ izidhilfe beim Sterbewilligen selbst ${ }^{41}$. Dieser führt die den Tod verursachende Handlung im Bewusstsein der tödlichen Wirkung selbständig aus $^{42}$. In der Regel betrifft die Suizidhilfe solche Fälle, in denen dem Suizidwilligen ein tödliches Betäubungsmittel verschrieben oder bereitgestellt wird $^{43}$. Die Suizidhilfe ist gemäss dem Sondertatbestand ${ }^{44}$ des Art. 115 als „Verleitung und Beihilfe zum Selbstmord" nur strafbar, wenn der Täter einen anderen zur versuchten oder vollendeten Selbsttötung aus „selbstsüchtigen Beweggründen" verleitet (Anstiftung) oder ihm dazu Hilfe leistet (Gehilfenschaft) ${ }^{45}$. Die von den Schweizer Suizidhilfeorganisationen durchgeführte Hilfe zur Selbsttötung blieb bis anhin straflos, weil ihnen keine selbstsüchtigen Motive nachgewiesen werden konnten ${ }^{46}$.

Um einen besonderen Fall der Suizidhilfe handelt es sich beim eingangs thematisierten Sterbetourismus. Da es sich dabei nicht um Fremdtötungen, also um Sterbehilfe, sondern um Suizidhilfe im vorerwähnten Sinne handelt, wäre es richtiger von „Suizidtourismus“ zu sprechen, sofern man nicht ohnehin auf die Anlehnung an den Begriff „Tourismus“ verzichten möchte ${ }^{47}$.

Der AE-StB 2005 sieht in § 215a AE-StB - ähnlich der Schweizer Regelung - die Einführung eines neuen Straftatbestands der „Unterstützung einer

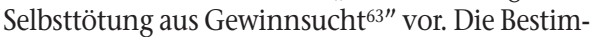
mung zielt auf die Tätigkeit von Suizidhilfeorganisationen wie Dignitas-Deutschland (DignitaTe). Gemäss dem AE-StB 2005 wäre geschütztes Rechtsgut einer solchen Norm „die Ausnutzung der Notlage eines tödlich kranken Menschen, die bei Gewinnsucht sozialethisch verwerflich ist"48. Diese als „misslungen” kritisierte Formulierung49 dürfte der Schwierigkeit geschuldet sein, überhaupt ein tragfähiges Rechtsgut namhaft zu machen. Lediglich sozialethische Verwerflichkeit also: nicht rechtsgutverletzendes Verhalten - vermag jedenfalls die Kriminalisierung gewinnsüchtigen Verhaltens kaum zu rechtfertigen ${ }^{50}$.

\section{Juli 2000: Der Bundesrat ist für eine Regelung passiver und indirekt aktiver Sterbehilfe}

Im Jahr 1994 reichte Nationalrat VICTOR RUFFY eine Motion ein, mit der er den Bundesrat ersuchte, einen Entwurf für einen neuen Art. 115 
vorzulegen. Der Motionär verlangte eine neue Regelung der Sterbehilfe-Bestimmungen, die der Situation unheilbar kranker, am Ende ihres Lebens stehender Menschen besser Rechnung trägt. Zwischen 1997 und 1999 setzte das EJPD eine aus Fachleuten verschiedener Fachrichtungen zusammengesetzte Arbeitsgruppe „Sterbehilfe“ ein, die beauftragt wurde, die in diesem Bereich sich stellenden komplexen Fragen abzuklären ${ }^{51}$.

\section{Direkte aktive Sterbehilfe}

Mehrheitlich sprach sich die Arbeitsgruppe „Sterbehilfe" dafür aus, die Fälle besser zu regeln, in denen Leiden eines todkranken Patienten mit herkömmlichen Mitteln nicht mehr gelindert werden können und er selber zu sterben wünscht. Für das Leisten von Sterbehilfe in solchen extremen und eher seltenen Fällen schlug die Mehrheit der Arbeitsgruppe ${ }^{52}$ vor, angesichts stark verminderter Schuld einen obligatorischen Strafbefreiungsgrund in Absatz 2 von Art. 114 ("Tötung auf Verlangen") vorzusehen:

„Hat der Täter eine in ihrer Gesundheit unheilbare beeinträchtigte, kurz vor dem Tod stehende Person getötet, um sie von unerträglichen und nicht behebbaren Leiden zu erlösen, so sieht die zuständige Behörde von der Strafverfolgung, der Überweisung an das Gericht oder der Bestrafung $a b^{\prime \prime 53}$.

Dieser neue Absatz 2 sollte also den bisherigen Art. 114 ergänzen (nicht ersetzen!), so dass - wie bisher - beabsichtigt war, vorauszusetzen, dass der Täter einen Menschen auf dessen „ernsthaftes und eindringliches Verlangen“ getötet und er selbst aus „achtenswerten Beweggründen” gehandelt hat (vgl. Art. 114). Der vorgeschlagene 2. Absatz sollte diesen Tatbestandsmerkmalen der aktuellen Gesetzesfassung noch besondere Elemente hinzufügen, die die Schuld des Täters stark vermindert erscheinen lassen. Diese sollten nach dem Mehrheitswillen der Arbeitsgruppe das Vorliegen einer unheilbaren zum Tode führenden Gesundheitsschädigung sein sowie der Umstand, dass der Täter im Bestreben gehandelt hat, unerträglichen und nicht behebbaren Leiden ein Ende zu setzen. Die zwingende Strafbefreiungsklausel hätte nicht nur für Medizinpersonal gegolten, weil auch Angehörige mit dem Sterbewunsch des Patienten genauso konfrontiert werden könnten wie ein Arzt. Ausserdem wäre sonst ein Mediziner von der Strafe befreit, während sich ein teilnehmender Angehöriger im Widerspruch dazu wegen Vorliegens einer vorsätzlichen und rechtswidrigen - aber straffreien - Haupttat des Arztes trotzdem der Gehilfenschaft (oder Anstiftung) hätte strafbar machen können. In subjektiver Hinsicht sollte es dem Täter - so der Gesetzesvorschlag der Arbeitsgruppe - darauf ankommen, die unerträglichen körperlichen oder seelischen Leiden $\mathrm{zu}$ beenden.

Der Bundesrat lehnte die Einführung dieses Strafbefreiungsgrundes als gesetzliche Regelung der direkten aktiven Sterbehilfe im Jahr 2000 ab. Er führte aus, dass selbst eine sehr restriktiv formulierte Ausnahme von der Strafbarkeit der direkten aktiven Sterbehilfe, wie sie von der Mehrheit der Arbeitsgruppe „Sterbehilfe" vorgeschlagen wurde, einer Lockerung des Fremdtötungsverbots gleich käme, so dass ein in der christlichen Kultur der Schweiz tief verankertes Tabu gebrochen würde. Auch im strafrechtlichen Schrifttum wird der Bedarf nach einer Novellierung des Tatbestandes der Tötung auf Verlangen als gering eingeschätzt.

\section{Indirekte aktive Sterbehilfe}

Übereinstimmend - und nicht bloss mehrheitlich - sprach sich die Arbeitsgruppe "Sterbehilfe“ dafür aus, die indirekte aktive und die passive Sterbehilfe im Strafgesetzbuch als ,erlaubte Handlungen“ innerhalb der Tötungsdelikte als Art. 114 oder Art. $115^{\text {bis }} \mathrm{zu}$ regeln.

Die indirekte aktive Sterbehilfe, also die Verabreichung schmerzlindernder Mittel mit lebensverkürzender Wirkung, sollte als Rechtfertigungsgrund normiert werden. Die Mitglieder der Arbeitsgruppe waren der Ansicht, dass dieser nicht nur dann zur Anwendung kommen solle, wenn der Arzt eine Verkürzung des Lebens des Patienten lediglich für möglich hält, sondern auch dann, wenn diese Folge so gut wie sicher sei. Allerdings müsse die Todesfolge als blosse Nebenwirkung des vom Täter eigentlich verfolgten Hauptziels (Leidenslinderung) erscheinen.

Im Unterschied zur direkten aktiven Sterbehilfe hat sich der Bundesrat im Jahr 2000 für eine ausdrückliche Regelung der indirekten aktiven Sterbehilfe im Strafgesetzbuch ausgesprochen. Zur Begründung führte er an, dass diese Form der Sterbehilfe schon heute zulässig sei und es deshalb Aufgabe des demokratisch legitimierten Gesetzgebers sei, sie im Gesetz zu regeln. Die Materie könne nicht allein der medizinischen Wissenschaft überlassen bleiben. Der Bundesrat führte weiter aus, dass es auch aus Gründen der Rechtssicherheit und der Rechtsgleichheit erforderlich sei, dass der Gesetzgeber das geschriebene Recht den Tatsachen anpasse.

\section{Passive Sterbehilfe}

Ebenso übereinstimmend kam die Arbeitsgruppe „Sterbehilfe” zum Schluss, dass auch die passive Sterbehilfe in Form einer gesetzlichen Regelung als rechtmässige Handlung anerkannt werden müsse. Als den am einfachsten zu regelnden Fall erachtete sie denjenigen, in dem eine mündige und urteilsfähige Person den Arzt - oder einen anderen Sorgepflichtigen - bittet, auf lebensverlängernde Massnahmen zu verzichten. Für diesen Fall müsse im Gesetz klar gesagt werden, dass derjenige, welcher diese Bitte des Kranken erfüllt, keine Tötung verübt und zwar unabhängig davon, ob der Patient dem Tode tatsächlich nahe ist oder nicht. Die Arbeitsgruppe führte weiter aus, dass im Falle einer mündigen, aber urteilsunfähigen Person der Verzicht auf lebensverlängernde Massnahmen als rechtmässig gelten solle, wenn er dem mutmasslichen aktuellen Willen der betroffenen Person entspreche. Zur Feststellung dieses hypothetischen Willens sei als objektives Kriterium auf die medizinische Prognose und in subjektiver Hinsicht auf frühere Willensäusserungen des Patienten abzustellen, die dieser in Form einer Patientenverfügung oder in mündlichen Äusserungen gegenüber Anghörigen oder medizinischem Personal bekundet hat. Für den Fall von unmündigen oder entmündigten urteils(un)fähigen Personen mochte sich die Arbeitsgruppe nicht definitiv zu einer wünschbaren Regelung äussern. Bei einer unmündigen urteilsfähigen Person sei zwar deren Wille massgebend, doch stelle sich auch hier - wie bei Urteilsunfähigen die Frage nach der Rolle des gesetzlichen Vertreters.

Der Bundesrat ist im Jahr 2000 der Arbeitsgruppe "Sterbehilfe” in ihrer Auffassung gefolgt und hat ebenfalls die Notwendigkeit einer Gesetzesregelung zur passiven Sterbehilfe betont. Die Sterbehilfe betreffe das Leben und damit das höchste Rechtsgut überhaupt. Daher sollte - so der Bundesrat - die Festlegung der Grenzen zwischen erlaubter und nicht erlaubter Tötung - wie bei der indirekten aktiven Sterbehilfe - vom demokratisch legitimierten Gesetzgeber vorgenommen und nicht den medizinischen Wissenschaften überlassen werden. Der Bundesrat führte weiter aus, dass eine klare strafgesetzliche Regelung auch im Fall der passiven Sterbehilfe den Vorteil böte, Rechtsgleichheit und Rechtssicherheit zu gewährleisten ${ }^{54}$.

\section{Zwischenergebnis}

Festzuhalten ist insoweit, dass sich der Bundesrat noch im Jahr 2000 für eine ausdrückliche strafgesetzliche Regelung der indirekten aktiven und der passiven Sterbehilfe ausgesprochen hat, ohne sich dabei jedoch auf einen konkreten Gesetzesvorschlag der Arbeitsgruppe „Sterbehilfe” stützen zu können. Gleichzeitig betonte er die bestehende Strafbestimmung über die direkte aktive Sterbehilfe (Art. 114) nicht ändern zu wollen, hob jedoch hervor, dass es alle Möglichkeiten der Palliativmedizin und -pflege auszuschöpfen gelte.

Seinen eigenen Bericht ${ }^{55}$ als Antwort auf die "Motion Ruffy" leitete der Bundesrat dem Parlament im Juli 2000 zu. Darin wird mit Rücksicht auf die soeben resümierten Argumente eine Änderung der Strafbestimmungen zur Sterbehilfe verlangt, die der Situation unheilbar kranker, am Ende ihres Lebens stehender Personen besser Rechnung trägt. Angesichts der Bedeutung dieser Frage wünschte der Bundesrat, dass die Diskussion über die Problematik der Sterbehilfe auch im Parlament weiter geführt wird. Im Folgenden wird der Blick um weitere sechs Jahre nach vorne - also auf das Jahr 2006 - gerichtet, um nachzu- 
vollziehen welche Entwicklung die dargelegte Positionierung des Bundesrates zum Thema „Sterbehilfe" in der Zwischenzeit genommen hat.

\section{Mai 2006: Der Bundesrat ist gegen eine Regelung passiver und indirekt akti- ver Sterbehilfe}

Am 3. Oktober 2001 reichte Nationalrat Guido ZäcH im Nationalrat die Motion „Sterbehilfe. Gesetzeslücke schliessen statt Tötung erlauben" ein. Der Bundesrat wird darin aufgefordert, Gesetzesänderungen auszuarbeiten und dem Parlament als Botschaft zu unterbreiten, um die Gesetzeslücken betreffend die indirekte aktive Sterbehilfe und die passive Sterbehilfe zu schliessen. In der Begründung wurde - wie zuvor vom Bundesrat selbst - darauf hingewiesen, dass diese beiden Formen der Sterbehilfe aus Gründen der Rechtssicherheit und Rechtsgleichheit im Gesetz zu regeln seien. Da jedoch der Bericht der Arbeitsgruppe „Sterbehilfe” auf konkrete Gesetzesvorschläge verzichtet habe, sei eine weitere Diskussion dringend geboten und es erscheine angebracht, dem Bundesrat den Auftrag zur Weiterarbeit zu erteilen, mit dem klaren Ziel, konkrete Lösungsmöglichkeiten auszuarbeiten ${ }^{56}$. Am 11. April 2003 hat auch die Kommission für Rechtsfragen des Ständerats im Ständerat die Motion „Sterbehilfe und Palliativmedizin" eingereicht. Darin wird der Bundesrat ebenfalls beauftragt, Vorschläge für eine gesetzliche Regelung der indirekten aktiven und der passiven Sterbehilfe zu unterbreiten und zudem Massnahmen zur Förderung der Palliativmedizin zu treffen ${ }^{57}$. Mit Datum vom 16. Juni 2005 reichte ausserdem die Freisinnig-demokratische Fraktion die Motion „Expertenarbeiten zum Thema Sterbehilfe" ein. Mit ihr wird der Bundesrat ebenso aufgefordert, die im EJPD ursprünglich einmal begonnenen Expertenarbeiten für einen Bericht bezüglich der Regelung der Sterbehilfe wieder aufzunehmen und bis Dezember 2005 dem Parlament die entsprechende Grundlage zur Verfügung zu stellen ${ }^{58}$. Auch (weite) Teile des neueren strafrechtlichen Schrifttums ${ }^{59}$ anerkennen die Regelungsbedürftigkeit der Sterbehilfe im Strafgesetzbuch ${ }^{60}$.

Zur Beurteilung des gesetzgeberischen Handlungsbedarfs setzte das EJPD eine verwaltungsinterne Arbeitsgruppe ein und beauftragte diese einen Bericht zum gesetzgeberischen Handlungsbedarf auf Bundesebene bezüglich der indirekten aktiven und der passiven Sterbehilfe, der Suizidhil$f e$ und des so genannten Sterbetourismus sowie zu möglichen Massnahmen zur Förderung der Palliativmedizin zu verfassen. Da nach Art. 123 Abs. 1 BV der Bund im Bereich der klassischen (Straf-) Rechtsgüter, wozu unzweifelhaft das menschliche Leben zählt, die abschliessende Gesetzgebungskompetenz besitz ${ }^{61}$, sind strafrechtliche Regelungen auf kantonaler Ebene ausgeschlos$\operatorname{sen}^{90}$. Den vom 24. April 2006 datierenden Bericht, der den gesetzgeberischen Handlungsbe- darf aus „heutiger Sicht”62 beurteilt, hat der Bundesrat am 31. Mai 2006 zur Kenntnis genommen. Ihn hat der Bundesrat zur Grundlage seiner Empfehlung an das Parlament gemacht, weshalb er hier in den wichtigsten Punkten aufgegriffen wird.

\section{Direkte aktive Sterbehilfe}

Der von Seiten des EJPD an die verwaltungsinterne Arbeitsgruppe vergebene Auftrag bezog sich, was die unterschiedlichen Formen der Sterbehilfe betrifft (vgl. II. 1.), ausschliesslich auf die indirekte aktive und die passive Sterbehilfe. Die vom Bundesrat zur direkten aktiven Sterbehilfe im Jahr 2000 vertretene Auffassung (vgl. III. 1.) wurde mithin nicht in Frage gestellt. Der Bericht „Sterbehilfe und Palliativmedizin” hält in seinen "Schlüssen" lediglich fest, dass die direkte aktive Sterbehilfe verboten ist.

\section{Indirekte aktive Sterbehilfe}

In Bezug auf die indirekte aktive Sterbehilfe stellt der Bericht fest, dass der Gesetzgeber im Strafgesetzbuch explizit regeln könnte, unter welchen Voraussetzungen eine Schmerzbehandlung mit lebensverkürzender Nebenwirkung straflos ist. Eine herausragende Rolle müsse dabei die ärztliche Aufklärungspflicht spielen, da die Folgen einer Schmerztherapie für den medizinischen Laien nicht ohne Weiteres offensichtlich seien. Denn die Abwägung der voraussichtlichen Leidensminderung und der damit einhergehenden Lebensverkürzung könne nur mit Hilfe medizinischen Wissens vorgenommen werden ${ }^{94}$.

\section{Passive Sterbehilfe}

Auch die explizite Straflosigkeit der passiven Sterbehilfe könnte der Gesetzgeber dem Bericht „Sterbehilfe und Palliativmedizin" zufolge im Strafgesetzbuch regeln. $\mathrm{Zu}$ bedenken sei dabei zum einen, unter welchen Voraussetzungen eine rechtsgültige Entscheidung für einen Behandlungsverzicht oder -abbruch gegeben ist; beispielsweise wäre zu normieren, ob diese nur in Form einer schriftlichen Patientenverfügung oder auch noch während der ärztlichen Behandlung möglich ist. Zum anderen wäre die Frage zu beantworten, auf Grund welcher Kriterien über einen Behandlungsverzicht entschieden werden darf, wenn der Patient dazu selbst nicht mehr in der Lage ist. Da dieses sehr unterschiedliche Konstellationen betreffen könne, wäre es aus Sicht der Berichtverfasser angebracht, diese Details entweder im Zivilgesetzbuch oder in einem Spezialgesetz zu regeln.

\section{Das „Für” und „Wider” einer strafgesetz- lichen Regelung}

Der Bericht "Sterbehilfe und Palliativmedizin" sieht als Vorteil einer gesetzlichen Regelung der indirekt aktiven und der passiven Sterbehilfe, dass diese mehr Transparenz schaffen könnte. Derzeit bedürfe es einiger Rechtskenntnisse, um abzuschätzen, ob sich ein Behandlungsverzicht oder -abbruch (passive Sterbehilfe) oder eine Schmerzbehandlung mit lebensverkürzender Nebenwirkung (indirekte aktive Sterbehilfe) noch im Rahmen des Legalen bewegt. Die Schwierigkeit liege hierbei jedoch weniger darin, zu beurteilen, „ob" die indirekte aktive und die passive Sterbehilfe grundsätzlich strafbar sind oder nicht, sondern vielmehr darin, festzustellen, „wer wann aufgrund welcher Sachlage" darüber entscheiden darf oder gar muss.

Problematisch erscheint den Berichtverfassern, die indirekte aktive Sterbehilfe klar von der direkten aktiven Sterbehilfe abzugrenzen. Würde die lebensverkürzende Schmerztherapie im Strafgesetzbuch prinzipiell straflos gestellt, so wäre die Abgrenzung zur direkten aktiven Sterbehilfe offen, was nicht der Sinn einer gesetzlichen Regelung sein könne.

Als eindeutigen Nachteil bezeichnet der Bericht, dass eine Regelung der aktiven und passiven Sterbehilfe (im Strafgesetzbuch) einem hohen Risiko ausgesetzt wäre, unvollständig zu bleiben. Dieses resultiere daraus, dass aus Gründen der erhöhten Transparenz einerseits alle Fallkonstellationen und Patientenkategorien bedacht werden müssten, andererseits aber die Regelung flexibel genug formuliert sein müsste, um allen denkbaren Einzelfällen und den künftigen Entwicklungen der Medizin zu genügen.

Die Berichtverfasser kommen unter Berücksichtigung dessen zum Schluss, dass sich das Strafgesetzbuch nicht als „Gefäss” für eine detaillierte Normierung aller denkbaren Fälle der indirekten aktiven und passiven Sterbehilfe eignet. Das Strafgesetzbuch könne allenfalls den „Ausgangspunkt" einer ausserhalb dieses Gesetzes liegenden Detailregelung bilden. Die Folge wäre, so der Bericht „Sterbehilfe und Palliativmedizin”, dass im Strafgesetzbuch nichts Neues geregelt, sondern nur bestätigt würde, was nach heutiger Rechtslage auch schon legal ist, so dass - abgesehen von etwas mehr Transparenz - keine „spürbare Verbesserung" der Rechtssicherheit und Rechtsgleichheit zu erwarten wäre. Wollte man in einer gesetzlichen Regelung mehr ins Detail gehen, dann würde sehr wahrscheinlich nur verbindlich festgeschrieben, was derzeit infolge der Richtlinien der Schweizerischen Akademie der Medizinischen Wissenschaften (SAMW) bereits Praxis ist. Ein inhaltlicher Gewinn wäre damit folglich nicht verbunden. Letztere seien vielmehr - da am geltenden Recht orientiert - aktuell eine sinnvolle Entlastung des Gesetzgebers. Dieses zusammenfassend kommen die Berichtverfasser zum Ergebnis, dass bei der indirekten aktiven und bei der passiven Sterbehilfe kein Handlungsbedarf für den Bundesgesetzgeber besteht. 
Gestützt auf diese Erwägungen empfiehlt nunmehr auch der Bundesrat dem Parlament, auf eine Revision der einschlägigen Bestimmungen des Strafgesetzbuchs zu verzichten und revidiert damit seine im Jahr 2000 vertretene Auffassung grundlegend. Damals hatte sich der Bundesrat, gestützt auf den Bericht „Sterbehilfe”, noch deutlich für eine gesetzliche Regelung der indirekten aktiven und der passiven Sterbehilfe im Strafgesetzbuch ausgesprochen (vgl. III. 2. und 3.).

\section{De lege ferenda: Der Bundesrat erwägt für die Suizidhilfe eine Revision des Betäu- bungsmittelrechts}

Im Problemkreis der Sterbehilfe ist in der Schweiz in den letzten Jahren der so genannte „Sterbetourismus”, der - wie erwähnt - besser als „Suizidtourismus" bezeichnet wird, in den Mittelpunkt des öffentlichen Interesses gerückt. Der wesentliche Grund für das anhaltende Phänomen des Suizidtourismus ist - wie bereits einleitend beschrieben - die unterschiedliche gesetzliche Regelung (insbesondere) in den Nachbarländern (vgl. I). Aus der unterschiedlichen rechtlichen Situation ${ }^{64}$ heraus hat sich - so der Bericht „Sterbehilfe und Palliativmedizin" - der Sterbetourismus in der Schweiz entwickelt ${ }^{65}$. Der vorerwähnte Bericht macht verschiedene Vorschläge zum Umgang mit diesem Phänomen. Der Bundesrat will sich in dieser Angelegenheit zukünftig „mit einer allfälligen Revision des Betäubungsmittelrechts [...] befassen" ${ }^{\prime \prime}$.

\section{Revision des Suizidhilfe-Rechts}

Die Berichtverfasser sehen mit Blick auf die Regelung der Suizidhilfe in Art. 115 mehrere Missbrauchsgefahren. Zum einen sei es schwierig nachzuweisen, dass der Suizident bei Vornahme der Selbsttötung noch urteilsfähig war und nicht durch Nötigung, Drohung oder Täuschung unter Druck gesetzt worden ist. Der Nachweis der Urteilsfähigkeit sei zum Beispiel bei der Überprüfung der Glaubwürdigkeit eines ausländischen Arztzeugnisses nur auf dem Wege der kosten- und personalintensiven internationalen Rechtshilfe möglich. Zum anderen sei fraglich, ob die Suizidhilfeorganisationen ihre Tätigkeit tatsächlich uneigennützig, also ohne „selbstsüchtige Beweggründe" ausüben. Die Suizidhilfeorganisation DIGNITAS verfolgt nach eigenen Angaben keine kommerziellen Interessen, da die eingenommenen Gelder ausschliesslich zur Deckung der entstehenden Kosten verwendet würden. Die Mitglieder zahlen eine einmalige Eintrittsgebühr von 100 Fr. und danach einen im Ermessen des Mitglieds liegenden Jahresbeitrag von mindestens 50 Fr. Der Bericht „Sterbehilfe und Palliativmedizin” führt weiter aus, dass die Standortgemeinden und -kantone von Suizidwohnungen und -häusern um ihr Ansehen fürchten und der gute Ruf der Schweiz insgesamt dauerhaften Schaden nehmen könne.
Sofern die Ursache für mögliche Missbräuche von Art. 115 sowie für den Suizidtourismus und den damit befürchteten Imageschaden in der liberalen Schweizer Gesetzeslage gesehen wird, böte sich zunächst an, diese Ursache anzugehen. Das heisst, den Art. 115 zu verschärfen, indem die Straffreistellung mit folgendem Wortlaut auf „unorganisierte" Suizidhelfer beschränkt würde:

„Wer zwar nicht aus selbstsüchtigen Gründen, aber berufsmässig bzw. wiederholt Suizidhilfe leistet, macht sich strafbar"68.

Gemäss dem Bericht „Sterbehilfe und Palliativmedizin" würde mit einer solchen Regelung aber die Verantwortung allein den nicht einer Strafdrohung unterliegenden Angehörigen eines Sterbewilligen aufgebürdet, die diese aber vielfach gar nicht tragen können oder wollen. Die Regelung käme damit in vielen Fällen faktisch einem Verbot der Suizidhilfe gleich.

Ein generelles (ausdrückliches) Verbot der Suizidhilfe wäre als drastischste Form der Gesetzesverschärfung zwar ebenso denkbar, nach Einschätzung der Berichtverfasser aber politisch nicht konsensfähig. Auch eine Beschränkung der Straffreistellung (bei Handeln ohne selbstsüchtige Motive) auf Personen mit Schweizer Wohnsitz wäre wegen der Ungleichbehandlung von Personen mit Auslandswohnsitz kaum tragfähig. Dieses berücksichtigend kommen die Berichtverfasser in ihrer „Zusammenfassung” zum Schluss, dass eine Änderung der liberalen Regelung von Art. 115 nicht in Betracht zu ziehen ist ${ }^{69}$.

\section{Aufsichtsgesetz zur „Qualitätssicherung” in der Suizidhilfe}

Die Berichtverfasser sprechen sich auch gegen ein spezielles Aufsichtsgesetz für Suizidhilfeorganisationen aus. Grundsätzlich sei es denkbar, für Suizidhilfeorganisationen ein solches Gesetz zu schaffen, um sie der staatlichen Aufsicht zu unterstellen und zur Einhaltung bestimmter Sorgfaltskriterien zu verpflichten. Eine Bewilligung würde danach beispielsweise nur an solche Organisationen und Suizidhelfer vergeben, die sich verpflichten, die im Gesetz vorzusehenden Voraussetzungen zu erfüllen und das vorzusehende Prozedere vor, während und nach dem begleiteten Suizid einzuhalten. Damit könnte zwar, so der Bericht „Sterbehilfe und Palliativmedizin", eine "Qualitätssicherung für Suizidhilfeorganisationen und Suizidhelfer" geschaffen werden, doch fehle für ein solches Bundesgesetz in der Bundesverfassung (wohl) eine entsprechende Erlassgrundlage. Zudem sei eine Bürokratisierung der Suizidhilfe und - wegen der „Qualitätssicherung" - eine noch grössere „Sogwirkung” für das Sterben in der Schweiz zu befürchten ${ }^{70}$.

Gestützt auf diesen Bericht hat der Bundesrat dem Parlament empfohlen, auf den Erlass eines
Gesetzes über die Zulassung und Beaufsichtigung von Suizidhilfeorganisationen zu verzichten ${ }^{71}$.

\section{Suizidbeistandschaft in Ergänzung des Vor- mundschaftsrechts}

Das Problem der Bürokratisierung sehen die Berichtverfasser auch im Hinblick auf die Schaffung einer von Amtes wegen zuzusprechenden Suizidbeistandschaft. Hinter diesem Konzept verbirgt sich die Idee, dass eine von einer Suizidhilfeorganisation zu begleitende Selbsttötung vorgängig der Vormundschaftsbehörde gemeldet wird. Diese würde sodann von Amtes wegen dem Suizidenten einen Suizidbeistand zusprechen. Dieser hätte die Aufgabe abzuklären, wie tief der Todeswunsch tatsächlich ist und ob er dem freien Willen des Suizidwilligen entspricht. Ferner hätte der Suizidbeistand zu prüfen, ob die Suizidbegleitung an einem dafür geeigneten Ort stattfinden soll und ob die Hilfeleistung der Organisation uneigennützig erfolgt. Sofern diese Voraussetzungen gegeben wären, müsste der Beistand den Suizid bei der Vormundschaftsbehörde noch bewilligen lassen. Für eine solche gesetzliche Regelung wäre nach Einschätzung der Berichtverfasser in Art. 122 Abs. 1 BV zwar die notwendige Rechtssetzungskompetenz des Bundes gegeben, doch würden sich beim Suizidtourismus, der Personen ohne Schweizer Wohnsitz erfasst, spezielle Probleme hinsichtlich der Zuständigkeit der Schweizer Behörden für die Suizidbeistandschaft stellen ${ }^{109}$. In ihrer „Zusammenfassung” beurteilen die Berichtverfasser daher eine gesetzliche Regelung im Bereich des Vormundschaftsrechts als „untaugliches” Mittel, um Missbräuche in der Suizidhilfe zu verhindern ${ }^{72}$.

\section{Revision des Betäubungsmittelrechts}

Zu einer positiven Bewertung kommt der Bericht „Sterbehilfe und Palliativmedizin” hingegen mit Blick auf eine Ergänzung des Betäubungsmittelgesetzes (BetmG). In den meisten von Suizidhilfeorganisationen begleiteten Suiziden werde das tödlich wirkende Betäubungsmittel Natrium-Pentobarbital (NAP) eingesetzt. Wenn Suizidhelfer in Ausübung ihrer Tätigkeit Betäubungsmittel wie das NAP verwenden, dann seien sie nach geltendem Recht auf die Mitwirkung von zugelassenen Ärzten angewiesen, da nach Art. 10 BetmG nur die in Art. 9 BetmG genannten, zur Berufsausübung ermächtigten Ärzte zum Verordnen von Betäubungsmitteln befugt seien. Deshalb sei es erwägenswert, gerade im Betäubungsmittelgesetz eine Bestimmung aufzunehmen, in der die Verschreibung dieser und vergleichbarer Substanzen an zusätzliche (restriktivere) Voraussetzungen gebunden wird, die eine Art „Spezialaufsicht” für die unter Abgabe von Betäubungsmitteln begleiteten Suizide schaffen ${ }^{73}$. Die Berichtverfasser schlagen beispielsweise vor, den verschreibenden Arzt zu einer vertieften Überprüfung des Falles zu verpflichten und die Möglichkeit der Verschreibung von NAP ausdrücklich auf Terminalkranke 
zu beschränken. Zudem sei es sinnvoll, den Bezug, die Abgabe und die Anwendung von NAP speziell zu reglementieren. Nach geltendem Recht werde das NAP (vom Apotheker) zwar auf Rezept für eine bestimmte Person abgegeben, was anschliessend damit passiere, würde jedoch zumindest nicht vom verschreibenden Arzt kontrolliert. So bestehe das Risiko, dass das NAP in "falsche Hände” gerate. Die für die Änderung des Betäubungsmittelrechts notwendige Verfassungsgrundlage ist nach Auffassung der Berichtverfasser in Art. 118 Abs. 2 lit. a BV gegeben ${ }^{112}$, so dass sie zusammenfassend feststellen, dass eine Revision des Betäubungsmittelrechts „erwägenswert” wäre, um den „heutigen Realitäten” in der Suizidhilfe zu begegnen.

Der Bundesrat teilt - wie gesagt - diese Einschätzung und wird sich daher in seiner weiteren Arbeit mit einer entsprechenden Revision des Betäubungsmittelrechts befassen.

\section{Fazit: Standpunktbestimmung}

Im Bericht „Sterbehilfe und Palliativmedizin Handlungsbedarf für den Bund?" vom 24. April 2006, der von einer internen Arbeitsgruppe des EJPD in diesem Jahr dem Bundesrat zur Kenntnisnahme vorgelegt wurde, sind die Fragen nach einem Handlungsbedarf des Bundesgesetzgebers bei der indirekten aktiven und der passiven Sterbehilfe sowie bei der Suizidhilfe und beim so bezeichneten Sterbetourismus behandelt worden. Der Bericht der EJPD-Arbeitsgruppe „Sterbehilfe” vom März 1999 hat sich mit Blick auf einen gesetzgeberischen Regelungsbedarf sowohl zur passiven als auch zur indirekten aktiven Sterbehilfe und zur direkten aktiven Sterbehilfe geäussert. Der Bundesrat hat auf beide Berichte mit eigenen Stellungnahmen reagiert. Zusammengefasst ergeben sich unter Einbezug des strafrechtlichen Schrifttums folgende Standpunkte:

\section{Bericht der Arbeitsgruppe „Sterbehilfe” (1999)}

- Passive und indirekte aktive Sterbehilfe werden im Gesetz ausdrücklich geregelt.

- Die direkte aktive Sterbehilfe bleibt ausnahmslos rechtswidrig.

- Mehrheitlich spricht sich die Arbeitsgruppe dafür aus, dass die in Art. 114 geregelte direkte aktive Sterbehilfe (Tötung auf Verlangen) um einen Absatz 2 ergänzt wird, der im Falle einer Mitleidstötung in auswegloser Lage (Unheilbarkeit, Todesnähe, unerträgliche, nicht behebbare Leiden) eine obligatorische Strafbefreiung vorsieht bzw. auf die Durchführung eines Strafverfahrens verzichtet.

\section{Bericht der Arbeitsgruppe „Sterbehilfe und Palliativmedizin" (2006)}

- Bei der passiven und der indirekten aktiven Sterbehilfe besteht kein Handlungsbedarf des
Bundesgesetzgebers; das Strafgesetzbuch ist kein geeignetes Gefäss zur Regelung dieser beiden Formen von Sterbehilfe.

- Die direkte aktive Sterbehilfe bleibt ausnahmslos strafbar.

- Eine Ergänzung des Betäubungsmittelrechts ist erwägenswert, um die Suizidhilfe (und den Suizidtourismus) einer Art Spezialaufsicht zu unterstellen.

- Als Reaktion auf den Suizidtourismus wird eine Revision von Art. 115 abgelehnt; eine Beschränkung der Straffreistellung auf „unorganisiert” Handelnde oder auf Personen mit Schweizer Wohnsitz sowie ein generelles Verbot der der Suizidhilfe kommen nicht in Betracht.

- Eine spezielle Aufsichtsgesetzgebung zur Kontrolle der Suizidhilfe und des Suizidtourismus wird abgelehnt.

\section{Stellungnahmen des Bundesrats (2000/2006)}

a) Bericht des Bundesrats zum "Postulat Ruffy” (2000)

- Der Bundesrat spricht sich für eine ausdrückliche Regelung der passiven und indirekten aktiven Sterbehilfe aus.

- Der Bundesrat lehnt eine Ausnahme von der Strafbarkeit der direkten aktiven Sterbehilfe (Strafbefreiungsklausel gemäss einem neuen Abs. 2 von Art. 114) ab.

\section{b) Empfehlung des Bundesrats, gestützt auf den "Sterbehilfe-Bericht" (2006)}

- Der Bundesrat empfiehlt dem Parlament auf eine Revision der einschlägigen Bestimmungen des Strafgesetzbuchs zu verzichten; im Bereich der passiven, direkt aktiven und indirekt aktiven Sterbehilfe sind keine weiteren gesetzlichen Regelungen notwendig.

- Im Bereich des Suizidhilfe und des damit im Zusammenhang stehenden Suizidtourismus erwägt der Bundesrat eine Revision des Betäubungsmittelrechts, um die Voraussetzungen für die Verschreibung der verwendeten tödlich wirkenden Betäubungsmittel restriktiver zu fassen.

- Der Bundesrat empfiehlt dem Parlament auf den Erlass eines Gesetzes über die Zulassung und Beaufsichtigung von Suizidhilfeorganisationen zu verzichten.

\section{Zu Stellungnahmen aus dem neueren strafrecht- lichen Schriftum}

- Eine gesetzliche Regelung der passiven und indirekt aktiven Sterbehilfe im Strafgesetzbuch wird begrüsst.

- Überwiegend wird (wohl) abgelehnt, für die Tötung auf Verlangen (direkte aktive Sterbehilfe) im Fall einer Mitleidstötung in auswegloser Lage eine obligatorische Strafbefreiung bzw. den Verzicht auf die Durchführung eines Strafverfahrens vorzusehen (Art. 114 Abs. 2 neu). Teilweise wird eine Lockerung des Verbots aktiver Sterbe- hilfe mit Rücksicht auf den nahenden Tod und das Selbstbestimmungsrecht des Sterbenden sowie die Praxis in den Spitälern allerdings als „aufrichtiger” empfunden.

Der Verfasser ist Assistenzprofessor für Strafrecht am Institut für Strafrecht und Kriminologie (ISK) der Universität Bern und Mitarbeiter am Lehrstuhl für Strafrecht der Universität St. Gallen (HSG).

\section{Fußnoten}

1 Für die Anregung des Themas und die wertvollen Hinweise danke ich Prof. Dr. K.-L. Kunz. Für die Durchsicht des Manuskripts geht mein Dank an Prof. Dr. H. Vest.

2 Bericht „Sterbehilfe und Palliativmedizin - Handlungsbedarf für den Bund?" vom 24. April 2006 einer internen Arbeitsgruppe des EJPD [im Folgenden: Bericht (2006)], S. 32.

3 Bericht (2006), S. 34

4 Bericht (2006), S. 33.

5 Bericht (2006), S. 34, dort Fn. 75 .

6 Vgl. die „Tätigkeitsberichte“ von Dignitas unter: www.dignitas.ch und die Angaben zu „Dignitate Deutschland" unter: www.dignitas.ch.

7 Medienmitteilung des EJPD vom 05.07.2000 (www.bj.admin.ch); Bericht des Bundesrats zum Postulat Ruffy, „Sterbehilfe. Ergänzung des Strafgesetzbuches" (2000), S. $14 \mathrm{f}$.

8 Zur Entwicklung seit Mitte der 1970er Jahre vgl. Heine, in: Eser/Koch, Materialien zur Sterbehilfe, Eine internationale Dokumentation (1991), Schweiz, 592 ff.; Zur Entwicklung in den 1990er Jahren vgl. Riklin, Die strafrechtliche Regelung der Sterbehilfe, in: Holderegger, Das medizinisch assistierte Sterben (1998), 328 ff. Die Notwendigkeit, alle Möglichkeiten der Palliativmedizin und -pflege auszuschöpfen und zu fördern, hat der Bundesrat hingegen damals wie heute gesehen, sie wird hier deshalb nicht weiter problematisiert.

9 Die rechtspolitische Auseinandersetzung zur Sterbehilfe war auch Gegenstand der Beratungen des 66 . Deutschen Juristentags (DJT) im September dieses Jahres in Stuttgart.

10 Gesetzesentwurf einer interdisziplinär zusammengesetzten Arbeitsgruppe, darunter zahlreiche Mitglieder des Arbeitskreises deutscher, österreichischer und schweizer Strafrechtslehrer (Alternativ-Professoren), vgl. Schöch/Verrel, GA 2005, 553 ff.

11 Schwarzenegger, in: Niggli/Wiprächtiger (Hrsg.), Basler Kommentar zum Strafgesetzbuch, Bd. II (2003), vor Art. $111 \mathrm{~N} 20$.

12 Petermann, Sterbehilfe: Eine terminologische Einführung, in: Ders., Sterbehilfe.Grundsätliche und praktische Fragen - Ein interdisziplinärer Diskurs, St. Gallen 2006, 21, 27 ff.; Donatsch, Die strafrechtlichen Grenzen der Sterbehilfe, recht 2000, 141, 143; Kunz, Sterbehilfe: Der rechtliche Rahmen und seine begrenzte Dehnbarkeit, in: Donatsch/Forster/ Schwarzenegger (Hrsg.), Strafrecht, Strafprozessrecht und Menschenrechte, Festschrift für Stefan Trechsel zum 65. Geburtstag, Zürich 2002, 613, 618; Schwarzenegger, in: BSK-StGB (2003), vor Art. 111 N 26 (vgl. Fn. 18); Trechsel, Kurzkommentar StGB (1997), vor Art. $111 \mathrm{~N}$ 9; Heine, in: Eser/Koch, Materialien zur Sterbehilfe, Eine internationale Dokumentation 
(1991), Schweiz, 592, 595; Riklin, Die strafrechtliche Regelung der Sterbehilfe, in: Holderegger, Das medizinisch assistierte Sterben (1998), 328, 329; Schubarth, Kommentar zum StGB, Besonderer Teil, 1. Bd. (1982), Systematische Einleitung, N 38.

13 Tötung (Art. 111), Totschlag (Art. 113), Tötung auf Verlangen (Art. 114), vgl. Trechsel, Kurzkommentar StGB (1997), vor Art. $111 \mathrm{~N} 8$.

14 Kunz, FS-Trechsel (2002), 613, 627 (vgl. Fn. 19).

15 „namentlich aus Mitleid”, vgl. Art. 114.

16 Kunz, in: FS-Trechsel (2002), 613, 618; Riklin, in: Holderegger, Das medizinisch assistierte Sterben (1998), 328, 329 (vgl. zu beiden Fn. 19)

17 Die kürzeste Dauer der Gefängnisstrafe ist drei Tage. Wo das Gesetz nicht ausdrücklich etwas anderes bestimmt, ist die längste Dauer drei Jahre, vgl. Art. 36.

18 Die kürzeste Dauer der Zuchthausstrafe beträgt ein Jahr, die längste Dauer 20 Jahre. Wo das Gesetz es besonders bestimmt, ist sie lebenslänglich, vgl. Art. 35 .

19 Mit der Reform des Allgemeinen Teils des Schweizerischen Strafgesetzbuchs, die voraussichtlich zum 1. Januar 2007 in Kraft treten wird, werden auch die Vorschriften über die Zuchthaus- und Gefängnisstrafe revidiert (vgl. neu Art. 40). Infolge dessen wird in Art. 111 die vorsätzliche Tötung in Zukunft "mit Freiheitsstrafe nicht unter fünf Jahren bestraft" und die Tötung auf Verlangen (Art. 114) „mit Freiheitsstrafe bis zu drei Jahren oder Geldstrafe". Am dargelegten Verhältnis zwischen Art. 111 und Art. 114 (Privilegierung) ändert sich also nichts. Zur AT-Reform vgl. Hansjakob/Schmitt/Sollberger, Kommentierte Textausgabe zum revidierten Strafgesetzbuch (2004).

20 Schöch/Verrel, AE-StB 2005, GA 2005, 553, 583 f.

21 Neumann/Saliger, Sterbehilfe zwischen Selbstbestimmung und Fremdbestimmung - Kritische Anmerkungen zur aktuellen Sterbehilfedebatte, HRRS 89/2006, 280, 285.

22 Der wesentliche Unterschied zur direkten aktiven Sterbehilfe besteht darin, dass bei letzterer der Tod des Patienten das vom Täter gewollte Mittel ist, um ihn von seinen Leiden zu erlösen; hingegen ist bei der indirekten aktiven Sterbehilfe der rasche Eintritt des Todes nur die vom Täter nicht gewollte, aber bedachte und gebilligte Folge der zur Linderung der Leiden verabreichten Substanzen, vgl. Petermann (2006), 21, 32 ff. (vgl. Fn. 12); Donatsch, recht 2000, 141, 144; Kunz, in: FS-Trechsel (2002), 613, 618; Trechsel, Kurzkommentar StGB (1997), vor Art. 111 N 8; Riklin, in: Holderegger, Das medizinisch assistierte Sterben (1998), 328, 337; Schubarth, Kommentar zum StGB, Besonderer Teil, 1. Bd. (1982), Systematische Einleitung, N 40 (vgl. zu allen Fn. 12); Schwarzenegger, in: BSK-StGB (2003), vor Art. 111 N 5 (vgl. Fn. 11).

23 Riklin, in: Holderegger, Das medizinisch assistierte Sterben (1998), 328, 338.

24 Problematisch ist in diesem Zusammenhang, dass Art. $32 \mathrm{im}$ Zuge der (voraussichtlich) zum 1. Januar 2007 in Kraft tretenden Reform des Allgemeinen Teils des Schweizerischen Strafgesetzbuchs durch den neuen Art. 14 ersetzt werden wird, der Amtsund Berufspflichten nicht mehr als Rechtfertigungsgründe erwähnt. Begründet wird dies damit, dass Amts- und Berufspflichten keine selbständigen Grundlagen bilden können, um straftatbestandliches Handeln zu rechtfertigen, sondern nur Eingriffsbefugnisse verleihen, wenn dafür eine gesetzliche
Grundlage besteht, vgl. Hansjakob/Schmitt/Sollberger, Kommentierte Textausgabe zum revidierten Strafgesetzbuch (2004), Kommentierung zu Art. 14. 25 Dogmatisch ist diese Rechtfertigung problematisch, da die Pflicht zur Erhaltung des Lebens generell gewichtiger einzustufen ist als die Pflicht Schmerzen zu lindern, vgl. Donatsch, recht 2000, 141, 144 (vgl. Fn. 12).

26 Vgl. Ziff. 3.1 der SAMW-Richtlinie „Betreuung von Patientinnen und Patienten am Lebensende".

27 Kunz, in: FS-Trechsel (2002), 613, 618 f; Riklin, in: Holderegger, Das medizinisch assistierte Sterben (1998), 328, 338 (vgl. zu beiden Fn. 12).

28 Schwarzenegger, in: BSK-StGB (2003), vor Art. 111 N 25 (vgl. Fn. 11).

29 A.A. Kunz, in: FS-Trechsel (2002), 613, 619 (vgl. Fn. 19), der unabhängig von der ärztlichen Intention die medizinisch indizierte Leidensverminderung mit dem Risiko der Lebensverkürzung für objektiv gestattet hält.

30 Kunz, in: FS-Trechsel (2002), 613, 619 (vgl. Fn. 19); Schubarth, Kommentar zum StGB, Besonderer Teil, 1. Bd. (1982), Systematische Einleitung, N 40.

31 Donatsch, recht 2000, 141, 143; Kunz, in: FS-Trechsel (2002), 613, 620 (vgl. zu beiden Fn. 12).

32 Trechsel, Kurzkommentar StGB (1997), vor Art. 111 N 7; Petermann (2006), 21, 34 ff. (vgl. Fn. 19).

33 Riklin, in: Holderegger, Das medizinisch assistierte Sterben (1998), 328, 337 (vgl. Fn. 12).

34 A.A. Schwarzenegger, in: BSK-StGB (2003), vor Art. 111 N 24 (vgl. Fn. 18). Er sieht im Abbruch von Behandlungsmassnahmen ein aktives Tun und damit eine direkte aktive Sterbehilfehandlung.

35 Strafbarkeit wegen Körperverletzung nach Art. 123.

36 Kunz, in: FS-Trechsel (2002), 613, 621 (vgl. Fn. 19); Trechsel, Kurzkommentar StGB (1997), vor Art. 111 N 7; Schubarth, Kommentar zum StGB, Besonderer Teil, 1. Bd. (1982), Systematische Einleitung, N 43.

37 Der Bundesrat hat am 28. Juni 2006 die Botschaft zum Erwachsenenschutz verabschiedet. In der Neuregelung des Vormundschaftsrechts wird in Art. $370 \mathrm{ff}$. E-ZGB auch die Patientenverfügung geregelt. Nach den Vorstellungen des Bundesrats soll eine Patientenverfügung für den behandelnden Arzt grundsätzlich verbindlich sein. Neu wird auch das Vorgehen bei fehlender Patientenverfügung in den Art. 377 ff. E-ZGB geregelt, vgl. Vollenweider, Gesetzgebungskompetenz der Kantone im Bereich der passiven Sterbehilfe, Jusletter vom 11. September 2006 Rz. 35.

38 Zur Ergründung dieses Willens sind dem Patienten nahestehende Personen anzuhören.

39 Kunz, in: FS-Trechsel (2002), 613, 621 (vgl. Fn. 19); Schwarzenegger, in: BSK-StGB (2003), vor Art. $111 \mathrm{~N}$ 23 (vgl. Fn. 18); Heine, in: Eser/Koch, Materialien zur Sterbehilfe, Eine internationale Dokumentation (1991), Schweiz, 592, 595 f.; Riklin, in: Holderegger, Das medizinisch assistierte Sterben (1998), 328, 332 ff. (vgl. Fn. 19); Schubarth, Kommentar zum StGB, Besonderer Teil, 1. Bd. (1982), Systematische Einleitung, $\mathrm{N} 44 \mathrm{ff}$.

39 Schwarzenegger, in: BSK-StGB (2003), vor Art. 111 N 8 (vgl. Fn. 11)

40 Schöch/Verrel, AE-StB 2005, GA 2005, 560 ff., 573 ff.; Neumann/Saliger, HRRS 8-9/2006, 280, 281.

41 Donatsch, recht 2000, 141; Kunz, in: FS-Trechsel (2002), 613, 618; Riklin, in: Holderegger, Das medi- zinisch assistierte Sterben (1998), 328, 330 (vgl. zu allen Fn. 11).

42 Fehlt dieses Bewusstsein, z.B. bei mangelnder Urteilsfähigkeit, dann ist kein Suizid gegeben, sondern ggf. eine strafbare Fremdtötung.

43 Sie kann aber auch darin bestehen, dass der sterbewilligen Person lediglich psychische Unterstützung zu dem von ihr verfolgten Ziel geleistet wird.

44 Art. 115 regelt die Beteiligung an einer Selbsttötung abschliessend. Eine Strafbarkeit wegen Tötung durch Unterlassen (lebensrettender Massnahmen ab dem Zeitpunkt der Bewusstlosigkeit) wie im deutschen Recht, ist daher nicht möglich, vgl. Riklin, in: Holderegger, Das medizinisch assistierte Sterben (1998), 328, 331 (vgl. Fn. 19); Donatsch (Hrsg.), Schweizerisches Strafgesetzbuch (2004), Anmerkungen zu Art. 115.

45 Petermann (2006), 21, 37 ff.; Donatsch, recht 2000, 141, 143; Ders. (Hrsg.), Schweizerisches Strafgesetzbuch (2004), Anmerkungen zu Art. 115; Kunz, in: FSTrechsel (2002), 613, 617; Riklin, in: Holderegger, Das medizinisch assistierte Sterben (1998), 328, 330.

46 Bericht „Sterbehilfe und Palliativmedizin” (2006), S. 9; Neumann/Saliger, HRRS 8-9/2006, 280, 288.

47 Vgl. zur Typologie der Sterbe- und Suizidhilfe auch die insoweit nicht zitierte Lehrbuch-Literatur: Rehberg/Schmid/Donatsch, Strafrecht III, Delikte gegen den Einzelnen (2003), dort den „Exkurs: Sterbehilfe” in $\S 1$, S. 16 ff. sowie Stratenwerth, Schweizerisches Strafrecht, Besonderer Teil I: Straftaten gegen Individualinteressen (2003), § 1, N 36 ff., 49 ff.; Ders., Sterbehilfe, ZStrR 1978, $60 \mathrm{ff}$.

48 Schöch/Verrel, AE-StB 2005, GA 2005, 553, 582.

49 Neumann/Saliger, HRRS 8-9/2006, 280, 288 (vgl. Fn. 21).

50 Dies gilt auch für den im Gesetzesentwurf der Länder Saaarland, Thüringen und Hessen formulierten Straftatbestand der Geschäftsmässigen Förderung der Selbsttötung, vgl. den Entwurf eines Gesetzes zum Verbot der geschäftsmässigen Vermittlung von Gelegenheiten zur Selbsttötung vom 27.03.2006 (BR-Dr. 230/06).

51 Bericht des Bundesrats zum Postulat Ruffy, „Sterbehilfe. Ergänzung des Strafgesetzbuches" (2002), S. 2 sowie die Medienmitteilung des EJPD vom 29.04.1999 (www.bj.admin.ch)

52 Die Position der Minderheit macht medizinische, rechtliche und sozialethische Bedenken geltend, vgl. dazu den Bericht der Arbeitsgruppe „Sterbehilfe“ an das EJPD, März 1999, S. 37 ff.

53 Bericht der Arbeitsgruppe „Sterbehilfe“ (1999), S. 35; vgl. hierzu auch Kunz, in: FS-Trechsel (2002), 613, 623 (vgl. Fn. 19) und Donatsch (Hrsg.), Schweizerisches Strafgesetzbuch (2004), Anmerkung zu Art. 114.

54 Medienmitteilung des EJPD vom 05.07.2000 (www.bj.admin.ch).

55 Bericht des Bundesrats zum Postulat Ruffy, „Sterbehilfe. Ergänzung des Strafgesetzbuches" (2000).

56 Die Motion wurde am 11. Dezember 2001 vom Nationalrat angenommen und am 17. Juni 2003 vom Ständerat in Form eines Postulats beider Räte an den Bundesrat überwiesen, vgl. die Motion „Sterbehilfe. Gesetzeslücke schliessen statt Tötung erlauben" 01.3523.

57 Die Motion wurde vom Ständerat am 17. Juni 2003 und vom Nationalrat am 10. März 2004 angenom- 
men, vgl. die Motion „Sterbehilfe und Palliativmedizin" 03.3180

58 Zur Begründung wurde ausgeführt, dass es nicht nachvollziehbar sei, dass mit dem Wechsel an der Departementsspitze des EJPD zwischenzeitlich der bereits in Auftrag gegeben Expertenbericht zum Thema Sterbehilfe wieder gestoppt wurde, vgl. die Motion „Expertenarbeiten zum Thema Sterbehilfe" 05.3352 .

59 Vgl. z.B. Kunz, FS-Trechsel (2002), 613, 629: „Angesichts der grundsätzlichen Bedeutung und Schwere der lebensbeendenden Massnahmen ist an sich eine gesetzliche Regelung erwünscht, wenn nicht geboten", ebenso Donatsch, recht 2000, 141, 143 (zur passiven Sterbehilfe): „Eine gesetzliche Regelung der umschriebenen Verhaltensweisen wäre erwünscht"; Ders., recht 2000, 141, 144 (zur indirekten aktiven Sterbehilfe): „eine gesetzliche Regelung dieser speziellen Fallkonstellation [ist] angezeigt"; Riklin, in: Holderegger, Das medizinisch assistierte Sterben (1998), 328, 350 (zur passiven und indirekt aktiven Sterbehilfe): „In allen diesen Fällen gibt das geltende Strafrecht keine hinreichende Orientierung über das gebotene bzw. erlaubte Handeln. [...] All dies spricht für eine gesetzliche Regelung".

60 Im älteren Schrifttum ist noch die Meinung vorherrschend, wegen der Unbestimmtheit der Massstäbe und des Fehlens hinreichend präziser objektiver Kri- terien entziehe sich die Sterbehilfe einer näheren gesetzlichen Regelung, vgl. z.B.: Stratenwerth, Sterbehilfe, ZStrR 1978, 60, 76; Schultz, Die Würde des Patienten: Ein Rechtsproblem?, in: Schweizerische Monatsschrift für Zahnheilkunde (1980), 1107, 1112; Gunzinger, Sterbehilfe und Strafgesetz (1978), S. 187; Rehberg, Arzt und Strafrecht, in: Handbuch des Arztrechts (1994), S. 316.

61 Trechsel, Kurzkommentar StGB (1997), Art. 335 N 1; Vest, in: Ehrenzeller/Mastronardi/Schweizer/Vallender (Hrsg.), Die Schweizerische Bundesverfassung (2002), Art. 123 N 2; Wirächtiger in: Niggli/ Wiprächtiger (Hrsg.), Basler Kommentar, Bd. II (2003), Art. 333 N 5 sowie Art. 335 N 4.

62 Vollenweider, Gesetzgebungskompetenz der Kantone im Bereich der passiven Sterbehilfe, Jusletter vom 11. September 2006 Rz. 36. Verschiedene Kantone haben aber in ihren Gesundheitsgesetzen Bestimmungen zum Umgang mit Sterbenden aufgenommen. Zuletzt wurde 2006 im Kanton Thurgau eine Motion zur Regelung der passiven Sterbehilfe eingereicht, vgl. Dies., Rz. 3.

63 Ihre Verletzung zieht zwar „nur” berufsständische und nicht unmittelbar staatliche Sanktionen nach sich, doch sind die Richtlinien schon heute mittelbar juristisch relevant, weil sie den Gerichten als Massstab für das sorgfaltsgemäss gebotene ärztliche Handeln dienen, vgl. Kunz, in: FS-Trechsel (2002), 613,
616 sowie Riklin, in: Holderegger, Das medizinisch assistierte Sterben (1998), 328, 331 (vgl. zu beiden Fn. 19). $\mathrm{Zu}$ den für die Sterbhilfe relevanten standesrechtlichen Bestimmungen vgl. auch Arzt, Für Sterbehilfe relevante standesrechtliche Bestimmungen im Lichte der Gesamtrechtsordnung, in: Petermann (2006), $69 \mathrm{ff}$.

64 Zur Rechtslage in Frankreich, Österreich, Italien, Spanien, Portugal, Polen, Grossbritannien, Holland, Belgien und den USA vgl. den Bericht "Sterbehilfe und Palliativmedizin" (2006), S. 35 f. sowie Schwarzenegger, in: BSK-StGB (2003), vor Art. 111, N 34 ff..

65 Bericht (2006), S. 36.

66 Medienmitteilung des EJPD vom 31.05.2006 (www.bj.admin.ch).

67 Bericht (2006), S. 38 ff. (vgl. dort auch Fn. 94).

68 Bericht (2006), S. 43.

69 Bericht (2006), S. 52.

70 Bericht (2006), S. $44 \mathrm{ff}$.

71 Medienmitteilung des EJPD vom 31.05.2006 (www.bj.admin.ch).

72 Bericht (2006), S. 52

73 Das Verschreiben von NAP stellt bereits eine ärztliche Tätigkeit dar, der eine persönliche Untersuchung und eine medizinische Indikation vorauszugehen haben, vgl. hierzu auch Kunz, Medienmitteilung des EJPD vom 31.05.2006 (www.bj.admin.ch).
Wieso muss die Schweiz befürchten, dass Deutsche die dortige liberale Rechtspraxis ausnutzen und Organisationen wie Dignitate Deutschland so etwas wie einen Sterbetourismus organisieren könnten? Liegt es am Recht oder der Praxis. Eicken vermutet, dass es das Recht sei und nennt die Fallgruppe der erbetenen "Tötung durch fremde Hand", die in Deutschland bei Garanten (Ärzte und Angehörige) eher zu einer Strafbarkeit wegen Tötung durch Unterlassen führen könnte. Ein prominentes Beispiel ist die Verurteilung eines Arztes wegen versuchter mittelbarer Tötung durch Unterlassen in BGH St 40 257, weil er bei einer Koma-Patientin die Krankenschwester angewiesen hatte, die künstliche Ernährung einzustellen, diese aber über die Klinikleitung bewirkte, dass der korrekte Weg eingeschlagen wird. Dieser sieht vor, dass in Fällen ohne

- Vorsorgevollmacht oder

- Patientenverfügung (am besten also beides notariell beurkunden lassen)

- nach § 1904 BGB analog eine Betreuung erfolgt und der Wunsch nach Beendigung einer ärztlichen Behandlung
- das Vormundschaftsgericht genehmigt, um einen präventiven Grundrechtsschutz zu erreichen. Dann ist selbstbestimmtes Sterben legal.

Allerdings gab es zum Zeitpunkt der oben genannten Verurteilung eines etwas $z u$ informell agierenden Arztes und der spektakulären BGH-Entscheidung zum Betreuungsrecht noch einen heftig und ideologisch geführten Meinungsstreit unter den zuständigen OLGen in Zivilsachen. Dieser ist vom BGH am 17.03.2003 (XII ZB 2/03) entscheiden worden, so dass die Rechtslage klar ist und auf der Webseite des Bundesministeriums der Justiz alle nötigen Formulare für das selbstbestimmte Sterben eingesehen werden können. Damit können Patienten vorsorgen und Ärzte die sehr restriktive Sicht der Bundesärztekammer, die einen Behandlungsabbruch im Vorfeld "vor dem Einsetzen des Sterbevorgangs" für standeswidrig hält, überspielen. Aber PatientInnen müssen vor der Behandlung einer tödlichen Erkrankung bzw. vor dem Zeitpunkt, in dem sie hinfällig werden, zum Notar gehen und alles regeln. Offenbar ist dieses Verfahren zu wenig bekannt, zu aufwendig oder nicht alltagstauglich. Jedenfalls wird es eher vermieden. Dann aber ist in Deutschland die Rechtslage in der Tat schwierig für Pflegebedürftige, die noch nicht so weit sind, dass der Sterbevorgang unmittelbar bevorsteht (hier können ÄrztInnen eine sinnlose Behandlung einstellen), die aber dennoch keine weitere Behandlung mehr wünschen und aufgegeben haben, dies aber nicht gerichtsfest dokumentieren. Erschwerend hinzu kommt die weit verbreitete Unkenntnis ausgerechnet bei den Personen, die mit diesen pflegebedürttigen Menschen zu tun haben. $Z u$ viel Polemik, zu wenig Pragmatik beherrscht das Feld. Kundigen Betroffenen und kundigen Angehörigen fällt es nicht schwer einen Weg zu finden (auch ohne aufwendige Vorsorge der Pflegebedürftigen). Aber wie macht man sich kundig? Offenbar ist deshalb Dignitate Deutschland so beliebt und fürchtet die Schweiz deshalb um ihren guten Ruf als Ferienland in Guten Zeiten.

Monika Frommel 